\title{
Segurança do paciente: principais eventos adversos na Unidade Terapia Intensiva
}

\author{
Patient safety: main adverse events in the Intensive Therapy Unit
}

Seguridad del paciente: principales eventos adversos en la Unidad de Terapia Intensiva

Italo Everton Bezerra Barbosa ${ }^{1 *}$, Alicia Ribeiro Fonseca ${ }^{1}$, Emmanuele Neuza Moreira de Andrade ${ }^{1}$, Dandara Conceição Maklouf ${ }^{1}$, Meire Cristina Soares Ribeiro ${ }^{1}$, Antônio José Paulo da Silva Rodrigues $^{1}$, Yasmin Tainá Corrêa Laborda ${ }^{1}$, Vitória Diniz Bezerra Lúcio da Silva ${ }^{1}$, Felipe Chrystian de Figueiredo Lira ${ }^{1}$, Stephanie Sampaio de Sena Gomes ${ }^{1}$.

\section{RESUMO}

Objetivo: Apontar os principais erros e eventos adversos que acometem a segurança do paciente em uma Unidade Terapia Intensiva (UTI), em conjunto com as medidas preventivas, que visem garantir a segurança dos pacientes. Métodos: Constitui-se de um estudo do tipo descritivo, com abordagem qualitativa, através de uma Revisão Integrativa da Literatura (RIL). O levantamento de dados foi realizado entre os meses de novembro de 2020 e janeiro de 2021, na plataforma Scientific Electronic Library Online (SCIELO), utilizando os descritores em saúde: segurança do paciente AND unidade de terapia intensiva, sendo encontrados 127 artigos na totalidade e após a aplicação dos critérios de inclusão e exclusão, considerados oito artigos para compor este estudo. Resultados: Os principais erros e falhas que acometem os pacientes dentro de uma UTI estão relacionados principalmente em três categorias: assistência de enfermagem, aumento do tempo de permanência na UTI e a carga horária de trabalho excessiva. Em relação aos principais EA, os de maior prevalência foram: lesões por pressão, quedas e danos de cateteres vasculares. Considerações finais: Logo, as medidas preventivas ao EA visam diminuir riscos e possíveis complicações aos assistidos, sendo necessária à sua discussão e entendimento de forma ampla e educativa.

Palavras-chave: Eventos adversos, Segurança do paciente, Unidade terapia intensiva.

\begin{abstract}
Objective: To point out the main errors and adverse events that affect patient safety in an Intensive Care Unit (ICU), in conjunction with preventive measures, aimed at ensuring patient safety. Methods: This is a descriptive research, with a qualitative approach, through an Integrative Literature Review (RIL). The data collection was carried out during the month of January 2021, in the database of the Scientific Electronic Library Online (SCIELO), using the descriptors in health, patient safety AND intensive care unit, with 127 articles in total, after the application of the inclusion and exclusion criteria, articles related to the topic were considered for review. Results: It was noticed that the main errors and failures that affect patients within an ICU are mainly related to three categories: nursing care, increased length of stay in the ICU and excessive workload in relation to the main AE pressure injury, falls and damage to vascular catheters were more prevalent. Final considerations: Given this, it becomes extremely important to pay attention to preventive measures with a possible prevention valve, such as $\mathrm{AE}$-related complications.
\end{abstract}

Keywords: Adverse events, Patient safety, Intensive care unit.

${ }^{1}$ Centro Universitário FAMETRO (CEUNI-FAMETRO), Manaus - AM. *E-mail: italoeverton1998@gmail.com

PUBLICADO EM: 2/2021 


\section{RESUMEN}

Objetivo: Señalar los principales errores y eventos adversos que afectan la seguridad del paciente en una Unidad de Cuidados Intensivos ( $\mathrm{UCl}$ ), en conjunto con las medidas preventivas, encaminadas a garantizar la seguridad del paciente. Métodos: Se trata de una investigación descriptiva, con enfoque cualitativo, a través de una Revisión Integradora de la Literatura (RIL). La recogida de datos se realizó durante el mes de enero de 2021, en la base de datos de la Biblioteca Electrónica Científica Online (SCIELO), utilizando los descriptores en salud, seguridad del paciente $Y$ unidad de cuidados intensivos, con 127 artículos en total, después Con la aplicación de los criterios de inclusión y exclusión, se consideraron para revisión los artículos relacionados con el tema. Resultados: Se observó que los principales errores y fallas que afectan a los pacientes dentro de una $\mathrm{UCl}$ se relacionan principalmente con tres categorías: cuidados de enfermería, aumento de la estadía en la UCI y sobrecarga de trabajo en relación al EA principal Las lesiones por presión, las caídas y los daños en los catéteres vasculares fueron más frecuentes. Consideraciones finales: Ante esto, es de suma importancia prestar atención a las medidas preventivas con una posible válvula de prevención, como las complicaciones relacionadas con los EA.

Palabras clave: Eventos adversos, Seguridad del paciente, Unidad de cuidados intensivos.

\section{INTRODUÇÃO}

A expressão "segurança do paciente", tem sido bastante utilizada dentro da área da saúde atualmente, tornando-se uma das áreas temáticas mais prioritárias e inovadoras nas últimas décadas. Pode ser definida como $O$ ato ou ações que possibilitem evitar, prevenir e melhorar as possíveis complicações e eventos adversos (EA) na vida dos pacientes, que podem acontecer principalmente pelos profissionais de enfermagem, na tentativa de prestar uma assistência de qualidade (CORPOLATO RC, et al., 2019).

$\mathrm{Na}$ unidade de terapia intensiva (UTI) os EA são bastante preocupantes, tendo em vista uma maior necessidade de procedimentos invasivos e de alta complexidade, em conjunto com uma demanda maior de medicamentos de alta gravidade clínica, resultando assim em um risco maior ao paciente. Deste modo, as consequências ocasionadas pelos EA, podem resultar principalmente no aumento da permanecia hospitalar e no aumento da taxa de mortalidade dos pacientes (DUARTE SCM, et al., 2019).

Os EA são indesejados, inesperados e estão diretamente ligados aos cuidados de saúde que são prestados ao paciente naquela área hospitalar. Estima-se que aproximadamente um em cada 10 pacientes no mundo são vítimas de EA durante o tratamento e assistência prestada a sua enfermidade. Além disso, a falta de segurança e de conhecimento dos profissionais da saúde podem resultar em procedimentos errôneos, colocando assim em risco a vida dos pacientes (ORTEGA DB, et al., 2017).

Com o surgimento das novas tecnologias, houve um aumento da ocorrência de EA e falhas nos serviços assistenciais prestados à segurança do paciente. Isso se deve ao fato de que antigamente os tratamentos eram mais simples e seguros, com o avanço da tecnologia, as terapias se tornaram cada vez mais eficazes, porém mais perigosas e complexas, necessitando de uma atenção e habilidade maior dos profissionais da área da saúde (SERAFIM CTR, et al., 2017).

A UTI corresponde a um ambiente hospitalar que tem como finalidade a manutenção da vida em conjunto com a recuperação da saúde, munida de procedimentos de alta complexidade, visando a promoção, recuperação e estabilização do indivíduo que se encontra em estado crítico, por meio de métodos e estratégias especializadas e eficazes. Em virtude destas intervenções terapêuticas, as chances de ocorrência de algum EA ou erros são maiores (ALVES KTA, et al., 2016).

Quanto mais complexos as terapias e procedimentos, maior será o risco de possíveis EA. Isso pode estar relacionado principalmente a sobrecarga de trabalho e a falta de conhecimentos e habilidades para realização dos procedimentos, levando ao erro durante a assistência de enfermagem, fazendo com que haja um agravamento do quadro clínico do paciente (MINUZZI AP, et al., 2016). 
Algumas estratégias para avaliação e monitoramento dos pacientes críticos têm sido utilizadas para tentar assegurar a qualidade da assistência prestada aos pacientes, para tentar evitar e reduzir os incidentes ocasionados por EA. Na UTI são empregadas algumas escalas que possibilitam a mensuração do prognóstico, parâmetros clínicos e a demanda de cuidados (CUADROS KC, et al., 2017).

Podemos citar, como exemplo, a Acute Physiology and Chronic Health Evaluation II (APACHE II), que é utilizada para prever a mortalidade hospitalar e estimar a gravidade das doenças, bem como o Nursing Activities Score (NAS), que mensura a carga horária de trabalho da equipe de enfermagem, incluindo ações direcionadas ao cuidado direto e desenvolvimentos das atividades gerenciais (RIBEIRO GSR, et al., 2018).

A preocupação de não causar danos aos pacientes é observada desde o surgimento da medicina, quando foi manifestada por Hipócrates, que em sua obra "Notas sobre Hospitais", fazia relatos sobre estratégias de prevenção de erros, relacionados as condições de trabalhos e desempenhos assistenciais de saúde. Uma vez que a qualidade da assistência prestada ao paciente, além de refletir na sua segurança, reflete também na importância e preocupação que se tem com esse tipo de temática (RIBEIRO GSR, et al., 2018).

Existem duas abordagens envolvidas nos erros e EA. A primeira refere-se à insegurança dos profissionais de saúde, gerando descuido, negligência, desatenção e imprudência na assistência. E a segunda abordagem, que envolve o sistema, as instalações, equipamentos e a organização, que tornam mais suscetíveis às falhas ativas, facilitando assim o aparecimento de EA, erros e incidentes (OLIVEIRA AC, et al., 2016).

Diante de todos os pontos citados e associados às incidências de EA em uma UTI e suas implicações na assistência, o presente artigo objetivou reunir conhecimentos e pesquisas que estejam relacionados à segurança do paciente e os principais EA que acometem a UTI em conjunto com as medidas preventivas.

\section{MÉTODOS}

Trata-se de uma pesquisa do tipo descritiva, com abordagem qualitativa, utilizando a Revisão Integrativa da Literatura (RIL) como técnica, uma vez que permite o conhecimento sobre estudos científicos referentes ao tema estudado, levando em consideração a sua abordagem em anos distintos, impulsionando o aprendizado e a escrita científica (SOARES CB, et al., 2014). Este estudo teve como base a seguinte pergunta norteadora: Quais os principais EA acometem a segurança do paciente em uma UTI?

A revisão integrativa torna possível resumir o passado, seja ele teórico ou empírico, dando a possibilidade de fornecer uma compreensão mais ampla de um determinado evento ou situação, além de possibilitar a inclusão de diferentes estudos, com diferentes metodologias, ideias e conceitos (WHITEMORE KN, et al., 2005).

A pesquisa foi desenvolvida em cinco momentos, sendo o primeiro referente a identificação do tema que seria abordado; o segundo direcionado aos estabelecimentos, os critérios de elegibilidade e inelegibilidade; o terceiro voltado para a avaliação dos artigos que seriam incluídos na revisão; no quarto, a interpretação dos resultados obtidos; e por último, a síntese completa dos artigos selecionados.

A pesquisa foi realizada entre os meses de novembro de 2020 e janeiro de 2021, nas bases de dados Scientific Electronic Library Online (SCIELO), utilizando os seguintes descritores: segurança do paciente AND unidade de terapia intensiva. Os critérios de elegibilidade estabelecidos foram: publicações gratuitas, disponíveis na íntegra do banco de dados, nos idiomas português e inglês, publicados durante o período de 2015 a 2020, que estivessem relacionados ao objetivo desejado.

Os critérios de inelegibilidade foram: publicações indisponíveis na íntegra de forma gratuita, publicados nos anos anteriores ao ano de 2015 , fora dos idiomas português e inglês, e que não estavam relacionados ao objetivo da pesquisa.

Todos os artigos que se enquadraram nos critérios de elegibilidade foram lidos por meio de seus resumos ou de forma integral quando apresentaram incongruências, mas expressavam aproximação com o objetivo do estudo. Após isto, realizada a análise e seleção dos artigos que fazem parte deste estudo. A revisão dos 
artigos teve a participação e avaliação de 5 autores de maneira independente. Por fim, os resultados foram comparados com o objetivo da pesquisa com finalidade de redução de erros ou a exclusão por engano de estudos que pudessem compor esta pesquisa.

\section{RESULTADOS}

Utilizando os descritores "Segurança do Paciente AND Unidade de Terapia Intensiva" foram encontradas 127 publicações na íntegra. Ao adicionar o filtro dos idiomas inglês e português, o número reduziu para 94, adicionando o critério de artigos publicados no ano de 2015 a 2020, o número diminuiu para 74. Após a análise dos 74 artigos publicados, foram selecionados e incluídos para revisão 8 artigos, direcionados ao tema (Figura 1).

Figura 1 - Fluxograma das etapas da seleção dos artigos para revisão.

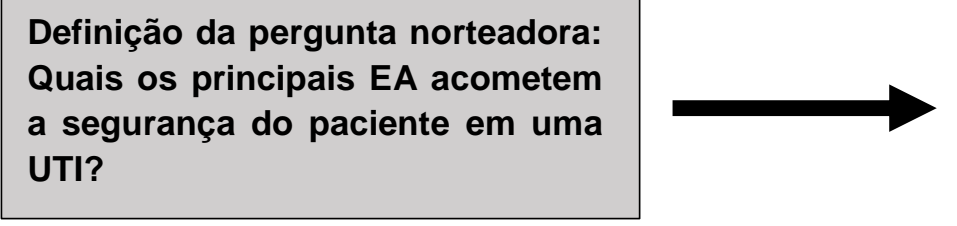

Busca na Base de Dados por meio do Scientific Electronic Library Online (SCIELO), com a seguinte combinação: Segurança do Paciente AND Unidade de Terapia Intensiva.
Encontrados: 127 artigos sem critérios.

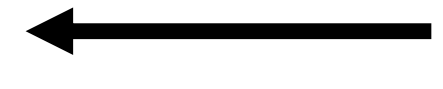

Descritores em ciências da saúde (DeCS): Segurança do Paciente e Unidade Terapia Intensiva.
Após a aplicação dos critérios de inclusão e exclusão: 74 artigos publicados direcionados ao tema.
Após a leitura dos resumos foram selecionados 8 artigos para revisão.

Fonte: Barbosa IEB, et al., 2021.

Em relação aos tipos de artigos, dos oito trabalhos escolhidos para revisão, três são de estudos descritivos (37,5\%), um retrospectivo (12,5\%), dois transversais (25\%), e dois prospectivo (25\%). Para melhor organização dos estudos selecionados, será apresentado uma síntese de levantamento dos estudos realizados conforme o ano de publicação, autor, título, objetivos, tipo de estudos, metodologias e publicação (Quadro 1). 
Quadro 1 - Artigos selecionados para revisão.

\begin{tabular}{|c|c|c|c|c|c|}
\hline $\mathbf{N}^{\circ}$ & Autor/Ano & Título & Objetivo & Estudo & Publicação \\
\hline 1 & $\begin{array}{l}\text { Ortega DB, et } \\
\text { al. (2017) }\end{array}$ & $\begin{array}{l}\text { Análise de eventos } \\
\text { adversos em pacientes } \\
\text { internados em unidade de } \\
\text { terapia intensiva }\end{array}$ & $\begin{array}{l}\text { Avaliar a incidência de } \\
\text { eventos adversos e associa- } \\
\text { los com a carga de trabalho } \\
\text { de enfermagem, o } \\
\text { dimensionamento da equipe } \\
\text { de enfermagem e o perfil de } \\
\text { gravidade do paciente. }\end{array}$ & $\begin{array}{l}\text { Transversal, } \\
\text { prospectivo } \\
\text { com } \\
\text { abordagem } \\
\text { quantitativa }\end{array}$ & $\begin{array}{l}\text { Acta Paul } \\
\text { Enferm. }\end{array}$ \\
\hline 2 & $\begin{array}{l}\text { Roque EK, et } \\
\text { al. (2016) }\end{array}$ & $\begin{array}{l}\text { Eventos adversos na } \\
\text { unidade de terapia } \\
\text { Intensiva: impacto na } \\
\text { mortalidade e no tempo } \\
\text { de internação }\end{array}$ & $\begin{array}{l}\text { Avaliar a ocorrência de } \\
\text { eventos adversos e o } \\
\text { Impacto deles sobre o tempo } \\
\text { de permanência e a } \\
\text { mortalidade na unidade de } \\
\text { terapia intensiva (UTI). }\end{array}$ & $\begin{array}{c}\text { Coorte } \\
\text { Prospectivo }\end{array}$ & $\begin{array}{l}\text { Cad. Saúde } \\
\text { Pública, Rio de } \\
\text { Janeiro, }\end{array}$ \\
\hline 3 & $\begin{array}{l}\text { Serafim CTR, } \\
\text { et al. (2017) }\end{array}$ & $\begin{array}{l}\text { Gravidade e carga de } \\
\text { trabalho relacionadas a } \\
\text { eventos adversos em UTI }\end{array}$ & $\begin{array}{l}\text { Analisar se o aumento da } \\
\text { gravidade do paciente e a } \\
\text { carga de trabalho de } \\
\text { enfermagem } \\
\text { relacionado a } \\
\begin{array}{l}\text { incidência de } \\
\text { está }\end{array} \\
\begin{array}{l}\text { Adversos (EA) em pacientes } \\
\text { críticos. }\end{array}\end{array}$ & $\begin{array}{c}\text { Coorte } \\
\text { Prospectivo }\end{array}$ & $\begin{array}{l}\text { Revista } \\
\text { Brasileira de } \\
\text { Enfermagem- } \\
\text { REBEn }\end{array}$ \\
\hline 4 & $\begin{array}{l}\text { Toffoletto MC, } \\
\text { et al. (2016) }\end{array}$ & $\begin{array}{l}\text { Fatores relacionados à } \\
\text { ocorrência de eventos } \\
\text { adversos em pacientes } \\
\text { idosos críticos }\end{array}$ & $\begin{array}{l}\text { Identificar os } r \text { fatores } \\
\text { relacionados a ocorrência de } \\
\text { eventos adversos em } \\
\text { pacientes idosos críticos } \\
\text { internados em UTI. }\end{array}$ & $\begin{array}{c}\text { Coorte } \\
\text { Retrospectivo }\end{array}$ & $\begin{array}{l}\text { Revista } \\
\text { Brasileira de } \\
\text { Enfermagem- } \\
\quad \text { REBEn }\end{array}$ \\
\hline 5 & $\begin{array}{l}\text { Minuzzi AP, et } \\
\text { al. }(2016)\end{array}$ & $\begin{array}{l}\text { Contribuições da equipe } \\
\text { de saúde visando à } \\
\text { promoção da segurança } \\
\text { do paciente no cuidado } \\
\text { intensivo }\end{array}$ & $\begin{array}{l}\text { Apresentar as } \\
\text { recomendações dos } \\
\text { profissionais de saúde de } \\
\text { uma Unidade de Terapia } \\
\text { Intensiva para a melhoria da } \\
\text { cultura de segurança do } \\
\text { paciente. }\end{array}$ & $\begin{array}{l}\text { Descritivo } \\
\text { Exploratório } \\
\text { com } \\
\text { abordagem } \\
\text { quantitativa }\end{array}$ & $\begin{array}{c}\text { Esc Anna } \\
\text { Nery -EEAN }\end{array}$ \\
\hline 6 & $\begin{array}{l}\text { Duarte SCM, et } \\
\text { al. (2019) }\end{array}$ & $\begin{array}{l}\text { Boas Práticas de } \\
\text { segurança nos cuidados } \\
\text { de enfermagem em } \\
\text { Terapia Intensiva } \\
\text { Neonatal }\end{array}$ & $\begin{array}{l}\text { Identificar a percepção dos } \\
\text { profissionais de enfermagem } \\
\text { sobre o erro humano nos } \\
\text { Cuidados de enfermagem na } \\
\text { Unidade de Terapia Intensiva } \\
\text { Neonatal. }\end{array}$ & $\begin{array}{l}\text { Descritivo } \\
\text { Quanti- } \\
\text { quantitativo }\end{array}$ & $\begin{array}{l}\text { Revista } \\
\text { Brasileira de } \\
\text { Enfermagem- } \\
\text { REBEn }\end{array}$ \\
\hline 7 & $\begin{array}{l}\text { Oliveira } A C \text {, et } \\
\text { al. }(2016)\end{array}$ & $\begin{array}{l}\text { Carga de trabalho de } \\
\text { enfermagem e ocorrência } \\
\text { de eventos adversos na } \\
\text { terapia intensiva: revisão } \\
\text { sistemática }\end{array}$ & $\begin{array}{l}\text { Buscar evidências sobre a } \\
\text { influência da carga de } \\
\text { trabalho de enfermagem na } \\
\text { ocorrência de EA (úlcera por } \\
\text { pressão, infecção, quedas ou } \\
\text { erros associados a } \\
\text { medicamentos) em pacientes } \\
\text { adultos internados em UTI. }\end{array}$ & Descritivo & $\begin{array}{c}\text { Rev Esc } \\
\text { Enferm USP }\end{array}$ \\
\hline 8 & $\begin{array}{l}\text { Cuadros KC, et } \\
\text { al. (2017) }\end{array}$ & $\begin{array}{l}\text { Ocorrência de incidentes } \\
\text { de Segurança do } \\
\text { Paciente e Carga de } \\
\text { Trabalho de } \\
\text { Enfermagem }\end{array}$ & $\begin{array}{l}\text { Identificar a relação entre a } \\
\text { carga de trabalho da equipe } \\
\text { de enfermagem e a } \\
\text { ocorrência de incidentes de } \\
\text { segurança dos pacientes } \\
\text { ligados aos cuidados de } \\
\text { enfermagem de um hospital } \\
\text { público no Chile. }\end{array}$ & $\begin{array}{l}\text { Transversal } \\
\text { analítica } \\
\text { quantitativa }\end{array}$ & $\begin{array}{l}\text { Rev. Latino } \\
\text { Am. } \\
\text { Enfermagem }\end{array}$ \\
\hline
\end{tabular}

Fonte: Barbosa IEB, et al., 2021. 
Ao analisar os artigos escolhidos para revisão, percebeu-se que os principais erros e falhas que acometem os pacientes em uma UTI, seja ela neonatal, pediátrica ou adulta, estão relacionadas principalmente a três categorias: assistência de enfermagem, aumento do tempo de permanência dos pacientes na UTI e o aumento da carga horária de trabalho. Ambas as situações estão diretamente ligadas ao aparecimento de lesão por pressão, quedas e danos por manejo nos cateteres vasculares, entre outros (Quadro 2).

Quadro 2 - Principais eventos adversos e erros que acometem o paciente em uma UTI.

\begin{tabular}{|c|c|c|c|}
\hline $\mathbf{N}^{\circ}$ & Eventos Adversos & Erros e Falhas & Publicações \\
\hline 1 & Lesão por pressão & Erros na Mudança de decúbito & Duarte SCM, et al. (2019) \\
\hline 2 & Quedas do leito & Esquecer de levantar a grade & Roque EK, et al. (2016) \\
\hline 3 & $\begin{array}{l}\text { Dano por manejo de cateteres } \\
\text { Vasculares }\end{array}$ & $\begin{array}{l}\text { Falha no procedimento de inserção e } \\
\text { manutenção do cateter }\end{array}$ & Oliveira AC, et al. (2016) \\
\hline 4 & Dano por manejo ventilatório & Falha na Intubação Orotraqueal & Ortega DB, et al. (2017) \\
\hline 5 & $\begin{array}{l}\text { Dano por manejo no cateter } \\
\text { gástrico }\end{array}$ & $\begin{array}{l}\text { Falha no Procedimento de inserção e } \\
\text { manutenção do cateter }\end{array}$ & Serafim CTR, et al. (2017) \\
\hline 6 & $\begin{array}{l}\text { Dano por manejo no cateter } \\
\text { urinário }\end{array}$ & $\begin{array}{l}\text { Falha no Procedimento de inserção e } \\
\text { manutenção do cateter }\end{array}$ & Roque El \\
\hline 7 & Infecção no trato Urinário & $\begin{array}{l}\text { Falha no Procedimento de inserção e } \\
\text { manutenção do cateter }\end{array}$ & $\mathrm{O}$ \\
\hline 8 & Infecção I & $\begin{array}{ll}\text { Falhas nas medidas } & \text { preventivas } \\
\text { relacionadas a infecção } & \end{array}$ & Du \\
\hline 9 & Pneumonia & $\begin{array}{l}\text { Falhas relacionadas a ventilação } \\
\text { mecânica }\end{array}$ & Or \\
\hline 10 & Sangramento & Falha no uso do anticoagulantes & Roque EK, et al. (2016) \\
\hline 11 & Equimose & Falha no uso do anticoagulantes & Duarte SCM, et al. (2019) \\
\hline 12 & Prurido & Falha no uso do analgésico opioide & Ortega DB, et al. (2017) \\
\hline 13 & Acatisia & Falha no uso do antiemético & Serafim CTR, et al. (2017) \\
\hline 14 & Bradicardia & Falha no uso de antiarrítmico & Roque EK, et al. (2016) \\
\hline 15 & Hematúria & Falha no uso de anticoagulantes & Duarte SCM, et al. (2019) \\
\hline 16 & Broncoespasmo & Falha no uso do antibiótico inalatório & Ortega DB, et al. (2017) \\
\hline 17 & Náusea/Vômito & Falha no uso do analgésico opioide e & Roque EK, et al. (2016) \\
\hline 18 & Hipertensão Arterial & Falha no uso de anti-hipertensivos & Ortega DB, et al. (2017) \\
\hline
\end{tabular}

Fonte: Barbosa IEB, et al., 2021.

\section{DISCUSSÃO}

Após a leitura, análise e interpretação dos artigos selecionados, os dados foram divididos quanto aos principais EA, erros e incidentes que acometem os pacientes em uma UTI, em conjunto das medidas recomendadas, que visem garantir a segurança dos pacientes, para que seja possível evitar novos erros futuramente.

Antes de abordarmos sobre os principais EA e erros que acometem o paciente em uma UTI, torna-se de extrema importância discutirmos as diferenças relacionadas entre os EA e os erros, para que a compreensão seja feita da forma correta. Erros são falhas prestadas na assistência envolvendo sua execução de maneira inadequada ou planejamento errado. Quando os erros geram danos aos pacientes, eles começam a ser caracterizados como EA, tornando-se ligado principalmente a morbimortalidade, acarretando ônus social e econômico, além do sofrimento por parte do paciente e dos familiares. Vale mencionar que os EA, podem ser evitados, uma vez que os mesmos são decorrentes das falhas geradas na própria assistência (SARAIVA S, 2015; CUNHA M, 2011).

\section{Principais EA e erros que acometem os pacientes em uma UTI}

Ortega DB, et al. (2017) avaliaram a incidência de EA e ver se os mesmos estavam relacionados com a carga horária de trabalho de enfermagem, associando ao perfil de gravidade do paciente. $O$ estudo foi realizado com uma abordagem quantitativa, participaram da pesquisa 304 pacientes internados em uma 
unidade de terapia intensiva, entre os meses de setembro e dezembro de 2013. Durante os quatro meses, ocorreram 39 eventos adversos, sendo com maior prevalência a lesão por pressão, dentre as causas de internações na UTI, as principais foram: doenças no trato respiratório e sepse.

No estudo de Roque EK, et al. (2016), procuraram avaliar a relação entre os EA e o tempo de permanência dos pacientes em uma UTI, realizou-se um estudo prospectivo, desenvolvido com 355 pacientes maiores de 18 anos, admitidos na UTI, no período de agosto a julho de 2012. Foram confirmados 324 EA, em 115 pacientes internados ao longo de um ano. Observou-se a taxa de incidência de 9,3\% de eventos adversos por dia. No estudo destacou-se uma série de EA, associados à assistência de enfermagem prestada aos pacientes, tendo como prevalência a lesão por pressão, correspondendo 59,3\% dos EA do estudo.

Em uma revisão sistemática da literatura, foi avaliada a relação da incidência de EA com a carga excessiva de trabalho da equipe de enfermagem em pacientes adultos em uma UTI. Os principais EA encontrados nos artigos selecionados foram: infecções, e lesão por pressão com maior prevalência (OLIVEIRA AC, et al., 2016).

Diante disso, em três das pesquisas selecionadas para revisão bibliográfica, o principal EA que acomete a segurança dos pacientes em uma unidade de terapia intensiva é a lesão por pressão, quedas e danos nos manejos de cateteres, estando associada principalmente a erros direcionados a assistência de enfermagem, carga horária de trabalho excessiva e longo tempo de internação, tornando o paciente mais suscetível a risco por infecções hospitalares.

\section{Influência e prevalência de fatores relacionados aos EA}

Sabe-se que a frequência dos EA que acometem o paciente em uma UTI, podem sofrer influência de diversos fatores, como gravidade, características da assistência prestada, doenças preexistentes, perfil e complexidade (DUARTE SCM, et al., 2019). No estudo de Ortega DB, et al. (2016), observou-se a prevalência de EA em pacientes idosos, embora outras pesquisas tenham evidenciado, em média, faixa etária menor que 65 anos.

Com relação à prevalência de sexo, o feminino apresentou $52 \%$ e o masculino $48 \%$. Referente a idade, a maior prevalência foi na faixa de 61 - 80 anos, com 35\% (ORTEGA DB, et al., 2017). Apesar dos dados apresentados, não foi observado estaticamente a ocorrência de EA, com características relacionadas à gravidade da complicação no paciente relacionados a (Idade e sexo) (ROQUE EK, et al., 2016).

\section{Relação entre os EA e o longo tempo de internação na UTI}

No estudo de Ortega DB, et al. (2017) o tempo médio de internação dos pacientes na UTI foi de 6 dias, diferente das internações clínicas que foram em média de 7,5 dias. Por outro lado, no estudo de Roque EK et al. (2016) o tempo mediano de permanência dos pacientes foi de 34 a 15 dias. Os EA mostraram ter relação com o tempo de internação, o aumento da permanência pode apresentar um maior índice de mortalidade aos pacientes que sofreram algum EA na UTI por um dano voltado para assistência prestada. A ocorrência de EA esteve associada principalmente ao tempo médio de 19 dias de permanência em uma UTI. Vale destacar que, o tempo de internação varia de acordo com a gravidade da patologia ou EA que acomete aquele paciente, podendo haver variação do tempo de internação. Porém, pacientes que ficam por um período maior de internação, estão mais suscetíveis a riscos de desenvolver complicações como infecções hospitalares e possíveis erros que pode levar ao surgimento de eventos adversos (ROQUE EK, et al., 2016).

\section{Medidas recomendadas para garantir a segurança do paciente em uma UTI}

As medidas recomendadas são direcionadas aos principais erros que acometem a segurança do paciente em uma UTI, pelos profissionais nas práticas assistenciais diárias. Será apresentado uma série de intervenções que tiveram como finalidade a prevenção e redução de riscos, garantindo ao paciente uma assistência segura e de qualidade, durante o seu atendimento hospitalar. As aplicações dessas recomendações podem servir de base para futuros planejamentos. Aprendizado organizacional e melhoria contínua: realizar um manual sobre a segurança do paciente; realizar capacitações dos funcionários 
periodicamente; elaborar protocolos operacionais padrão (POP); realizar aprimoramento aos novos funcionários, com a finalidade de corrigir e evitar erros de profissionais recém-admitidos (ROQUE EK, et al., 2016).

Percepção geral da segurança do paciente: atentar para o horário, dose e diluição correta dos medicamentos; focar no paciente; conscientizar profissionais, familiares e pacientes sobre riscos de contaminação; cuidado com a higienização do box e limpeza do ambiente; atentar-se para trocas de luvas e descarte correto de perfurocortantes; reduzir o fluxo de visitas para pacientes em estado críticos ou instáveis (DUARTE SCM, et al., 2019).

Lesão por pressão: planejamento de protocolos que visem diminuir o aparecimento de lesão por pressão nos pacientes; informar a equipe sobre a importância de realizar a mudança de decúbito; utilizar protetores para articulações e proeminência óssea; realizar a inspeção de pele e identificação de pacientes que possuem maior facilidade de desenvolver lesão por pressão (MINUZZI AP, et al., 2016). Retirada e manutenção de dispositivos terapêuticos: realizar higienização e trocar do dispositivo sempre que necessário; orientar os familiares e os pacientes sobre a manutenção dos dispositivos; orientar a equipe de enfermagem sobre as técnicas corretas de inserção e manuseio de dispositivos de cateter e sondas (ORTEGA DB, et al., 2017).

Quedas e medidas restritivas: sempre elevar as grades da cama; realizar campanhas sobre a vigilância e observação dos pacientes; adequar a infraestrutura da área hospitalar na UTI; aplicação de técnicas de contenção de movimento corretas; adicionar algodão ortopédico nos pulsos para não lesionar a pele do paciente (MINUZZI AP, et al., 2016). Abertura para comunicações: intensificar o diálogo e a comunicação de forma efetiva entre os profissionais e pacientes; realizar reuniões com os profissionais sempre que possível; realizar levantamentos dos principais EA que acometem a segurança do paciente e reforçar as medidas preventivas, promovendo discussões de casos clínicos (DUARTE SCM, et al., 2019).

Respostas não punitivas ao erro e gestão hospitalar: não punir os profissionais por EA acometidos aos pacientes; disponibilizar materiais de qualidade para os procedimentos, assim como sua quantidade; trocar as bombas de infusão sempre que necessárias; promover manutenção dos equipamentos e criar uma comissão responsável por medidas que visem manter a segurança do paciente (SERAFIM CTR, et al., 2017). Transferências internas e passagem de plantão: realizar a passagem do plantão de maneira correta; promover cuidados seguros relacionados a transferência dos pacientes; realizar passagem dos plantões para técnicos quando o enfermeiro estiver presente (MINUZZI AP, et al., 2016).

Pode-se observar que, as maiorias das recomendações são direcionadas aos profissionais assistenciais, que estão diretamente ligadas à sua capacitação, para o manuseio de equipamentos e dispositivos, utilização de sedativos, mudanças de decúbito, quedas e entre outros, conforme presente nos estudos (DUARTE SCM, et al., 2019). No estudo de Minuzzi AP, et al. (2016), foram levantadas situações para que seja possível diminuir os EA, dentre elas a ampliação do comprometimento, responsabilidade e cooperação entre os membros da hierarquia, para que assim seja possível ter uma boa comunicação e um clima agradável no trabalho. Diante disso, a maioria dos estudos selecionados para revisão apresentaram o mesmo padrão de informações, tanto nas questões referentes aos principais EA que acometem o paciente em uma UTI, mas como também em relação ao tempo de internação e as medidas preventivas, com a finalidade de evitar o acontecimento de erros que levem ao aparecimento de EA nos pacientes.

Convém mencionar que, os pacientes internados em uma UTI, estão mais vulneráveis a possíveis complicações hospitalares, tanto pelo seu estado crítico, mas como também pelas infecções ou erros assistenciais. Porém, algumas situações podem ainda ter uma influência maior e complicar ainda mais essa situação, como a passagem de plantão ou anotações nos prontuários, que muitas vezes pela falta de atenção, pode ocasionar erros e até mesmo EA.

Com base na análise dos artigos revisados, pode-se afirmar que todas as recomendações informadas foram direcionadas para que sejam utilizadas dentro da UTI, para que seja possível garantir a segurança do paciente, afim de evitar possíveis incidentes que venham impor a sua vida ou de terceiros em risco. Diante disso, os recursos de uma UTI, capacitação dos profissionais e a terapia medicamentosa, são apontadas como necessárias para que seja possível combater os EA e impedir erros aos pacientes. 


\section{CONSIDERAÇÕES FINAIS}

Diante de todos os dados apresentados, a ocorrência de EA ainda corresponde a um grave problema nos leitos de UTI. Nesse sentido, a busca por artigos que abordassem o mesmo tema, nos últimos cinco anos, contribuiu para essa afirmação. Com isto, é de extrema importância destacar as medidas preventivas, com a finalidade de prevenir as possíveis complicações relacionadas aos erros, sendo eles assistenciais, gerenciais ou até de longa permanência hospitalar. A maioria dos erros apresentados neste estudo estavam relacionados aos serviços assistenciais prestados pela equipe de enfermagem, por isso a importância de capacitar esses profissionais de forma correta e contínua, com a finalidade de evitar possíveis erros aos pacientes, bem como às técnicas e manuseios de equipamentos.

\section{REFERÊNCIAS}

1. ALVES KYA, et al. Segurança do paciente na terapia intravenosa na unidade de terapia intensiva. Revista de Pesquisa: Cuidado é Fundamental Online, [S.I.], 2016; 8(1): 3714-3724.

2. BARBOSA TP, et al. Práticas assistenciais para segurança do paciente em unidade de terapia intensiva. Acta Paul Enferm., 2014; 27(3): 243-8.

3. BRANCO A, et al. Education to prevent Ventilator associated pneumonia in Intensive Care Unit. Rev Bras Enferm. 2020; 73(6): e20190477.

4. CASTRO AF e RODRIGUES MCS. Audit of standardized precautionary and contact practices in the Intensive Care Unit. Rev Esc Enferm USP. 2019; 53: e03508.

5. CORPOLATO RC, et al. Standardization of the duty shift in a General Adult Intensive Care Unit. Rev Bras Enferm [Internet]. 2019; 72(Suppl 1): 88-95.

6. CAPUCHO HC, CASSIANI SHB. Necessidade de implantar programa nacional de segurança do paciente no Brasil. Rev. Saúde Pública, 2013; 47(4): 791-8.

7. CUADROS KC, et al. Patient Safety Incidents and Nursing Workload. Rev. Latino-Am. Enfermagem. 2017; 25: e2841.

8. DUARTE SCM, et al. Best Safety Practices in nursing care in Neonatal Intensive Therapy. Rev Bras Enferm. 2020; 73(2): e20180482.

9. LLAPA EO, et al. Assistência segura ao paciente no preparo e administração de medicamentos. Ver Gaúcha Enferm. 2017; 38(4): e2017-0029.

10. MINUZZI AP, et al. Contribuições da equipe de saúde visando à promoção da segurança do paciente no cuidado intensivo. Esc. Anna Nery [online]. 2016; 20(1): 121-129.

11. ORTEGA DB, et al. Análise de eventos adversos em pacientes internados em unidade de terapia intensiva. Acta paul. enferm. [online]. 2017; 30(2): 168-173.

12. OLIVEIRA AC, et al. Nursing workload and occurrence of adverse events in intensive care: a systematic review. Rev Esc Enferm USP. 2016; 50(4): 679-689.

13. OLIVEIRA JLC, et al. Interface between accreditation and patient safety: nursing team perspectives. Rev Esc Enferm USP. 2020; 54: e03604.

14. OLIVEIRA AEC, et al. Alarm fatigue and the implications for patient safety.Rev Bras Enferm [Internet]. 2018; 71(6): 3035-40.

15. PACHÁ HHP, et al. Pressure Ulcer in Intensive Care Units: a case-control study. Rev Bras Enferm [Internet]. 2018; 71(6): 3027-34.

16. RIBEIRO GSR, et al. Equipment failure: conducts of nurses and implications for patient safety. Rev Bras Enferm [Internet]. 2018; 71(4): 1832-40.

17. RIBEIRO AC, et al. Cultura de segurança do paciente: percepção dos enfermeiros em um centro de referência em cardiopneumologia.

18. SERAFIM CTR, et al. Severity and workload related to adverse eventsin the ICU. Rev Bras Enferm [Internet]. 2017; 70(5): 942-8.

19. SILVA R, et al. Visibilidade do transporte intra-hospitalar em unidade de terapia intensiva: estudo descritivo. Rev Gaúcha Enferm. 2017; 38(3): e2017-0048

20. SASSAKI RL, et al. Interruptions and nursing workload during medication administration process. Rev Bras Enferm. 2019; 72(4):

21. SPECHT AM, et al. Incidência de quedas em uma coorte de adultos críticos: motivo de preocupação? Rev Gaúcha Enferm. 2020; 41(esp): e20190167.

22. TOFFOLETTO MC, et al. Factors associated with the occurrence of adverse events in critical elderly patients. Rev Bras Enferm [Internet]. 2016; 69(6): 977-83.

23. SOARES CB, et al. Revisão integrativa: conceitos e métodos utilizados na enfermagem. Rev. esc. enferm. USP, São Paulo, 2014; 48(2): 335-45. 